\title{
Demonstration of 40kV TEM Diffraction of Graphite for Structural Analysis
}

\author{
Andrew Barnum ${ }^{1}$ and Jun Jiao ${ }^{1,2}$ \\ 1. Physics Department, Portland State University, Portland, OR 97201, USA \\ 2. Mechanical and Materials Engineering, Portland State University, Portland, OR 97207, USA
}

Rapid structural assessment of newly synthesized nanomaterials is of tantamount importance both to exploratory researchers and industries seeking integration with consumer products. Most nanomaterials are susceptible to radiation damage during observation in the transmission electron microscope (TEM), causing propagation of dislocations, altering crystal phases, and destruction of the lattice structure through amorphization. Lowering the observation voltage in the TEM can result in less damage, though at the expense of decreased resolution, for many nanomaterials [1]. For microscopes not equipped with aberration correctors, such as the Tecnai F-20 used here, an information space roughly equivalent to $200 \mathrm{kV}$ operation can be achieved at $40 \mathrm{kV}$ through diffraction, greatly increasing the length of time for observation and the certainty of the observed structural details.

To ensure stable observation, nanographite was dissolved in a $0.75 \%$ formvar solution in chloroform, based on known dissolution rates [2]. Two drops from a Pasteur pipette were placed in rapid succession onto the surface of a beaker filled with distilled water and the chloroform allowed to dry - water tension naturally pulled the drying formvar into an electron transparent film. The films were pulled off and treated according to standard methods afterward [3]. During prolonged observation, the films proved highly susceptible to heating damage; evaporation of $3 \mathrm{~nm}$ of amorphous carbon onto the beam exit side alleviated the problem.

Observation of these samples was carried out first at $200 \mathrm{kV}$, where the $0.34 \mathrm{~nm}$ graphite lattice was easily observed. During prolonged observation, the expected degradation of the crystal structure occurred. In diffraction mode, this degradation was readily apparent as a continuous loss in intensity within the diffraction rings in less than a minute. Repeating the observation at $40 \mathrm{kV}$ allowed an equal quantity of primary diffraction reflections to be observed, but with observation times exceeding 20 minutes. Intensity integration of the diffraction rings was accomplished by first applying a logarithmic function to the pixel values of the images, followed by background subtraction [4]. The indexed intensity plots, presented alongside the diffraction patterns in Figures 1a and 1b, demonstrate that little information is lost in reciprocal space during the transition to a $40 \mathrm{kV}$, despite the inability to image lattice fringes in real space. One surprising benefit of lower voltage was an increase in the ability to resolve small peaks. Due to a decrease in camera saturation for otherwise equivalent gun and beam settings, the (100) and the much smaller (111) peak are well separated at $40 \mathrm{kV}$; at $200 \mathrm{kV}$, deconvolution is required to fully separate and index the (111) contribution.

The method presented here for $40 \mathrm{kV}$ diffractive observation promises to provide excellent structural resolution for many beam sensitive materials. Though requiring both careful alignment of the microscope and delicate sample preparation, this method permits increased observation times for carbon-based compounds, ranging from the inorganic, such as those described above, 
as well as organic crystals; all are especially well suited to analysis at this low voltage, without accompanying concerns for radiation-related structural changes.

\section{References:}

[1] Stoger-Pollach, M. "A Short Note on How to Convert a Conventional Analytical TEM into an Analytical Low Voltage TEM." Ultramicroscopy, 2014, 94-97.

[2] Marcus, Yizhak. "Solubility of C60 Fullerene." Journal of Physical Chemistry B, no. 13 (2001): 2499-2506.

[3] http://www.2spi.com/catalog/grids/lacey-carbon-formvar.html. Accessed Jan 15th, 2015.

[4] Vainshtein, B. K. Structure Analysis by Electron Diffraction. Burlington: Elsevier Science, 1964.
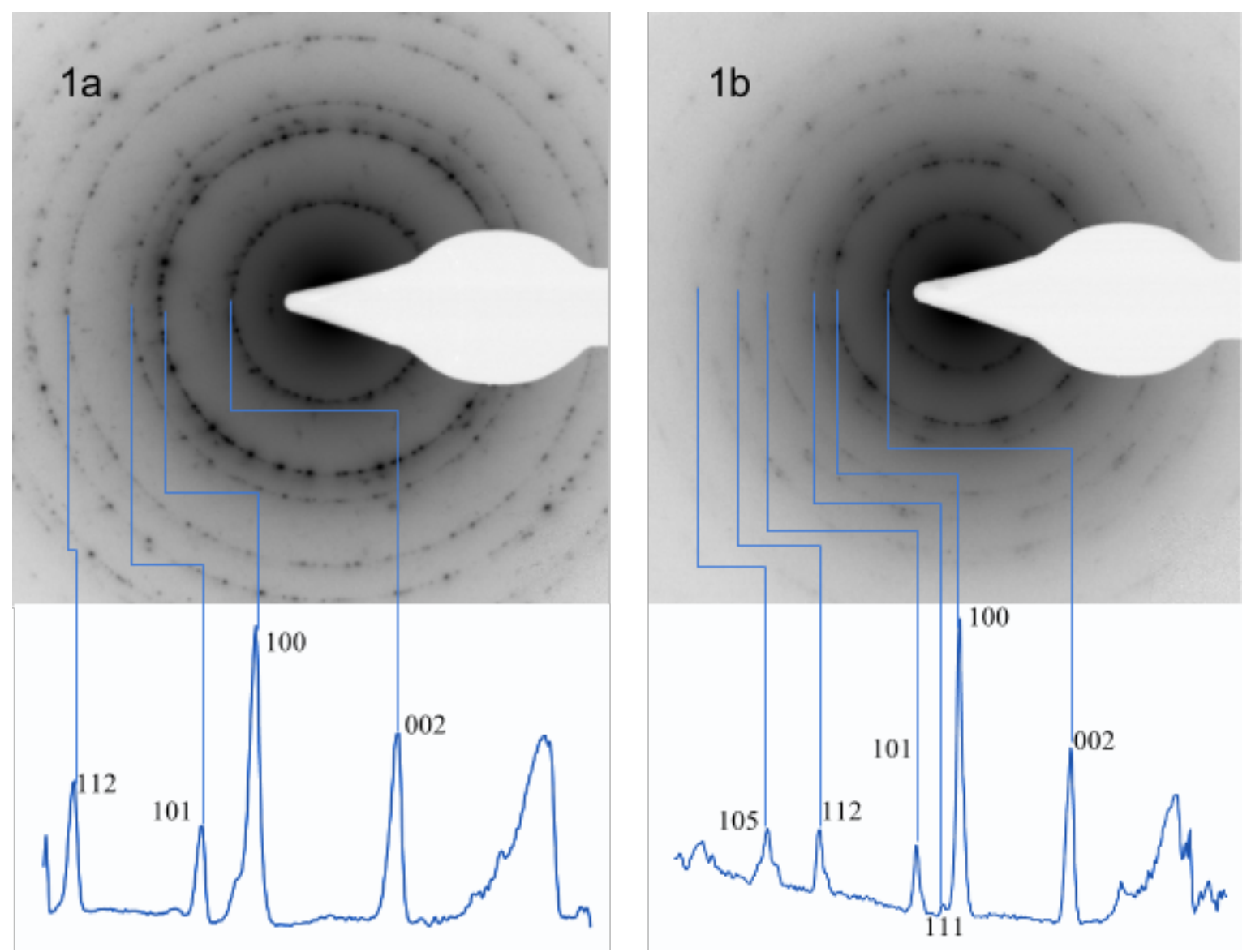

Figure 1: (a) Nanographite diffraction pattern acquired at 200kV. (b) Nanographite diffraction pattern acquired at $40 \mathrm{kV}$. Both patterns have been contrast inverted for ease of examination. 